Ann. Biol. anim. Bioch. Biophys., 1979, 19 (3 B), 975-981.

\title{
Déterminisme de la dualité de l'excrétion fécale chez le lapin : effets de l'ablation totale du côlon proximal
}

\author{
F. GALLOUIN, Germaine DEMAUX, H. LE BARS \\ Institut National Agronomique \\ 16, rue Claude Bernard, 75005 Paris.
}

Summary. The effect of surgical removal of the colon ascendens on soft and hard fecal production in rabbits.

When the colon ascendens was surgically removed from 7 New Zealand rabbits, soft fecal production and the practice of coprophagy were definitively abolished. After colectomy, the rabbits lost weight for about 20 days and then began to eat and grow. The initial weight was reached after about 2 months. Post-mortem examination showed no enlargement of either the fusus coli or the caecum. In experiments designed to determine the effect of colectomy on fecal production, fecal samples were analyzed for nitrogen and water. The feces produced had a chemical composition intermediate between those of hard and soft feces. According to Bonnafous and Raynaud, these results would suggest that the colon ascendens is indispensable in rabbits for soft fecal, production.

\section{Introduction.}

Dans un précédent travail (Gallouin ef al., 1978) nous avons cherché si le Lapin était capable de produire des cæcotrophes et des fèces dures en l'absence de cæcum. Nous avons réalisé la mise hors circuit de l'organe en posant une ligature autour du cæcum juste en aval du sacculus rotondus. Dans ces conditions, le Lapin n'excrète plus qu'un seul type de fèces à teneur en acides gras volatils et en azote intermédiaire entre les fèces normales et les cæcotrophes. Quinze à vingt jours après l'opération la dualité de l'excrétion réapparaît. Ceci est de nouveau possible car nous constatons à l'autopsie réalisée après sacrifice des Lapins qu'il s'est formé un « néocæcum » par dilatation du carrefour iléo-cæco-colique. Bonnafous et Raynaud (1967) ont insisté les premiers sur le rôle fondamental du côlon proximal dans la fabrication des cæcotrophes. Ces auteurs réalisent des colectomies partielles $(7$ à $8 \mathrm{~cm})$ limitées à la portion aborale du côlon proximal ; le Lapin excrète alors des fèces de type intermédiaire entre les cæcotrophes et les fèces dures. Mais ce phénomène est transitoire : la dualité de l'excrétion fécale réapparaît 15 jours après l'opération. Ces auteurs interprètent ce retour à la normale par le fait que la partie voisine du segment enlevé s'allonge et permet ainsi la vicariance. Herndon ef Hove (1955) puis Bacquès et Perref (1971) après cæcectomie 
n'avaient pas observé un phénomène analogue au niveau du cæcum. Par contre ces mêmes auteurs avaienł observé que le côlon proximal se développait et que le Lapin sans cæcum pouvait encore émettre deux types de fèces sans toutefois que la cæcotrophie ne réapparaisse. Il existe donc deux phénomènes bien distincts : l'élaboration des cæcotrophes d'une part, et leur ingestion ou cæcotrophie d'autre part.

Dans notre travail nous avons cherché à savoir quel était le rôle global du côlon proximal dans ces phénomènes en réalisant l'exérèse totale de cette portion du gros intestin. Dans ces conditions, la vicariance constatée par Bonnafous et Raynaud est-elle encore possible ? Si elle ne l'est plus et si le côlon proximal est bien le siège de l'élaboration des cæcotrophes on doit voir disparaître à la fois la production ef l'ingestion de ce type de fèces. Dans ce cas quels seront la croissance et le comportement alimentaire du Lapin?

\section{Matériel et méthodes.}

Neuf Lapins mâles de race Néo-Zélandaise ont été opérés. Les animaux reçoivent une injection de Nozinan (ND) à la dose de $15 \mathrm{mg} / \mathrm{kg}$ en sous-cutané, $30 \mathrm{~min}$. avant l'anesthésie générale obtenue par une injection intra-veineuse de pentobarbital sodique $(25 \mathrm{mg} / \mathrm{kg}$ dilué 4 fois dans l'eau bidistillée). La laparotomie est pratiquée sur la ligne blanche, elle s'étend de l'ombilic au pubis. Le cæcum et le côlon sont extériorisés ef placés sur des compresses imbibées de sérum physiologique tiède. Les vaisseaux sanguins de la paroi du cæcum anastomosés avec ceux du côlon proximal sont ligaturés à la soie $4 / 0$ puis sectionnés. Les feuillets du mesentère sont incisés parallèlement au côlon proximal. Des pinces à forcipressure sont placées au niveau de la jonction cæco-colique et en amont du fusus coli. La colectomie est alors réalisée à l'aide du bistouri (30 cm environ). L'orifice cæcal étant de plus grand diamètre que celui du fusus coli, il est nécessaire d'en effectuer la réduction de façon à ce que les deux diamètres deviennent compatibles. Les deux conduits sont reliés entre eux à l'aide d'une sufure termino-ferminale réalisée à points séparés (soie 4/0. aiguille de section triangulaire). Les points traversent complètement les parois intestinales la suture est donc muco-muqueuse. L'étanchéité est contrôlée ainsi que la possibilité de transit, après élimination des pinces à forci-pressure. Une suspension aqueuse de bipénicillinestreptomycine ( $1 \mathrm{M} / 1 \mathrm{~g}$ ) est versée dans la cavité abdominale. Les plans musculaires et cutanés sont restaurés selon les techniques habituelles. Un pansement est solidement fixé jusqu'à la cicatrisation totale du plan cutané. Les animaux reçoivent une injection de sérum glucosé isotonique, de vitamine $C(20 \mathrm{mg} / \mathrm{kg})$ et de prednisolone $(1 \mathrm{mg} / \mathrm{kg})$. Les injections de corticoïdes et de vitamines $C$ sont renouvellées trois jours de suite.

Après l'opération les Lapins sont placés individuellement dans des cages à métabolisme permettant la récolte des excréta. Ils disposent ad libitum d'aliments composés (Extralabo C 17).

Nous avons suivi sur les animaux opérés, la consommation alimentaire ef l'évolution du poids corporel. Les excréta ont été ramassés et pesés à l'état frais, jour après jour, à la même heure. Après homogénéisation du prélèvement, une aliquote est lyophilisée et nous déterminons la teneur en matière sèche et en azote total : critères qui nous ont paru les mieux caractériser la nature de l'émission fécale (fèces ou cæcotrophes). Un jour par semaine, un carcan interdisant la pratique de la cæcotrophie, est 
placé autour du cou de chaque Lapin afin de savoir si la production des cæcotrophes persiste. Le rythme d'émission est en même temps étudié en recueillant les fèces d'heure en heure à l'aide d'un collecteur de fraction (Bezille, Gallouin et Le Bars, 1973):

Les Lapins n'ayant pas été opérés tous le même jour, nous avons rapporté tous les résultats au jour de l'opération pris comme jour de référence zéro.

\section{Résultats.}

Sur neuf Lapins opérés, deux n'ont pas supporté l'opération. Six des animaux restants, après une période de reprise et de maintien en bon état général pendant dix ou douze semaines, ont été sacrifiés en fin de matinée et autopsiés. Un Lapin (612) après une période de reprise satisfaisante a dû être sacrifié et autopsié 36 jours après l'opération car son état général déclinait.

A l'autopsie, nous n'avons jamais observé de régénération du côlon proximal. Le volume du cæcum nous a paru légèrement augmenté chez deux animaux, par contre le contenu cæcal était dans l'ensemble plus déshydraté que d'habitude. L'estomac des Lapins sacrifiés ne contenait pas de cæcotrophes bien que l'heure de l'autopsie corresponde à celle pendant laquelle ils réalisent le comportement de cæcotrophie.

La consommation alimentaire est reportée dans le tableau 1. Ce tableau représente les consommations moyennes journalières exprimées en grammes, la moyenne étant réalisée sur une semaine.

TABLEAU 1

Consommations moyennes journalières des Lapins durant les semaines suivant l'opération

\begin{tabular}{|c|c|c|c|c|c|c|c|}
\hline \multirow{2}{*}{$\begin{array}{c}\text { Temps } \\
\text { après opération } \\
\text { (semaines) }\end{array}$} & \multicolumn{7}{|c|}{ Lapins numéro : } \\
\hline & 587 & 590 & 603 & 611 & 606 & 609 & 612 \\
\hline $\begin{array}{r}1 \\
2 \\
3 \\
4 \\
5 \\
6 \\
7 \\
8 \\
9 \\
10 \\
11 \\
12\end{array}$ & $\begin{array}{r}- \\
\overline{75} \\
30 \\
28 \\
51 \\
63 \\
57 \\
65 \\
101 \\
- \\
-\end{array}$ & $\begin{array}{l}77 \\
44 \\
39 \\
38 \\
47 \\
96 \\
62 \\
52 \\
58 \\
98\end{array}$ & $\begin{array}{r}78 \\
53 \\
69 \\
48 \\
92 \\
106 \\
99 \\
110 \\
111 \\
110 \\
- \\
-\end{array}$ & $\begin{array}{r}- \\
46 \\
53 \\
97 \\
103 \\
112 \\
112 \\
110 \\
132 \\
120 \\
115 \\
-\end{array}$ & $\begin{array}{r}3 \\
42 \\
44 \\
70 \\
146 \\
107 \\
123 \\
108 \\
73 \\
- \\
-\end{array}$ & $\begin{array}{r}22 \\
25 \\
39 \\
83 \\
93 \\
73 \\
122 \\
151 \\
167 \\
- \\
-\end{array}$ & $\begin{array}{r}33 \\
36 \\
82 \\
108 \\
54 \\
- \\
- \\
- \\
- \\
-\end{array}$ \\
\hline
\end{tabular}

Nous observons que les consommations restent faibles pendant les quatre premières semaines après l'opération ( $40 \mathrm{à} 70 \mathrm{~g} /$ jour/animal), elle atteint $100 \mathrm{~g}$ et plus, au-delà de ce délai chez les animaux les plus jeunes mais reste faible chez les deux plus âgés (587 et 590). La consommation des opérés n'a jamais atteint le niveau de celle 
d'animaux de notre animalerie maintenus dans des conditions comparables $(180 \mathrm{~g} /$ jour $)$. Ce faible niveau de consommation n'empêche cependant pas une reprise de croissance des Lapins comme le montre la figure 1.

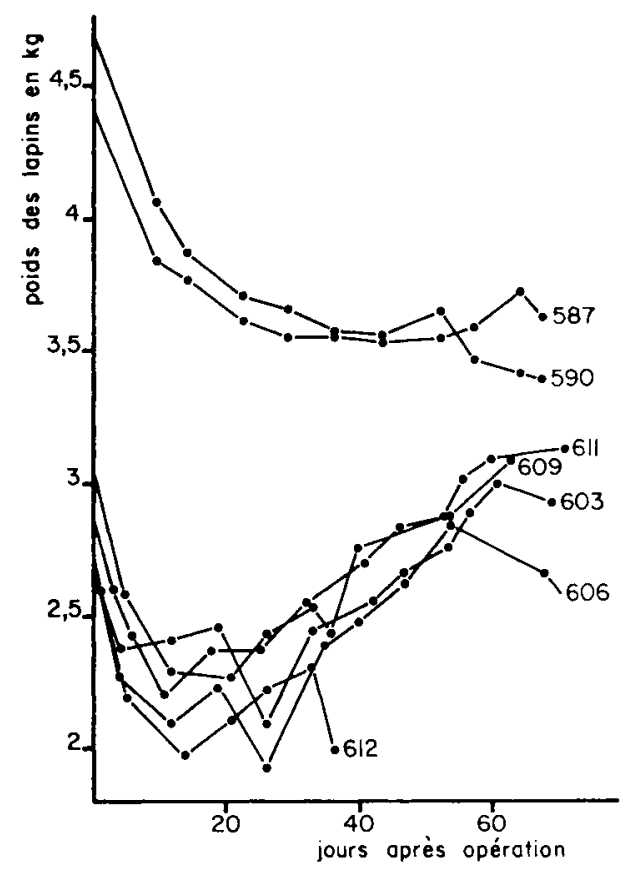

FIG. 1. - Evolution du poids de 7 Lapins après ablation totale du côlon proximal. La chute de poids se stabilise, pour tous les animaux, vers le 15e jours après l'opération. Les Lapins no 587 et 590 perdent du poids jusqu'au 20e jour puis cessent de maigrir. Par contre les Lapins nos 611, 603, 609,606 retrouvent leur poids initial en deux mois après une perte de 30 à 50 p. 100 .

La chute de poids est de l'ordre de 25 à 30 p. 100 du poids initial, la reprise s'annonce au cours de la $3 \mathrm{e}$ ou $4^{\mathrm{e}}$ semaine après l'opération chez les sujets les plus jeunes (603-606-609-611-612) et ceci avec une remarquable homogénéité des résultats, alors que chez les deux Lapins plus âgés (587-590) le poids se stabilise et ne revient pas au niveau antérieur.

En ce qui concerne les excréła, nous observons que le transit digestif est rétabli en général aprés $48 \mathrm{~h}$. La quantité produite est proportionnelle à la consommation. Les fèces émises ont un aspect uniforme de fèces dures hydratées. Ce type unique d'excréta a été le seul que nous ayons recueilli, même lorsque les lapins sont munis du carcan empêchant la cæcotrophie.

Dans la figure 2 nous avons représenté à l'aide de colonnes blanches les teneurs moyennes journalières en azote ef en matière sèche des émissions fécales des Lapins opérés. Pendant une période de 57 jours la composition en azote est très stable ef voisine de celle des fèces dures normales d'animaux maintenus dans les mêmes conditions d'élevage $(29,26 \pm 2,34 \mathrm{mg} / \mathrm{g} \mathrm{sec})$. La quantité de matière sèche contenue dans les excréta est intermédiaire entre celle des fèces dures normales 
51,6 p. 100 et celle de cæcotrophes normaux ( 31,7 p. 100). Cette quantité varie d'un jour à l'autre. Ces résultats indiquent que nous sommes en présence de fèces de type intermédiaire entre les cæcotrophes et les fèces dures en ce qui concerne la richesse en eau, mais que leur teneur en azote est celle de fèces dures normales.

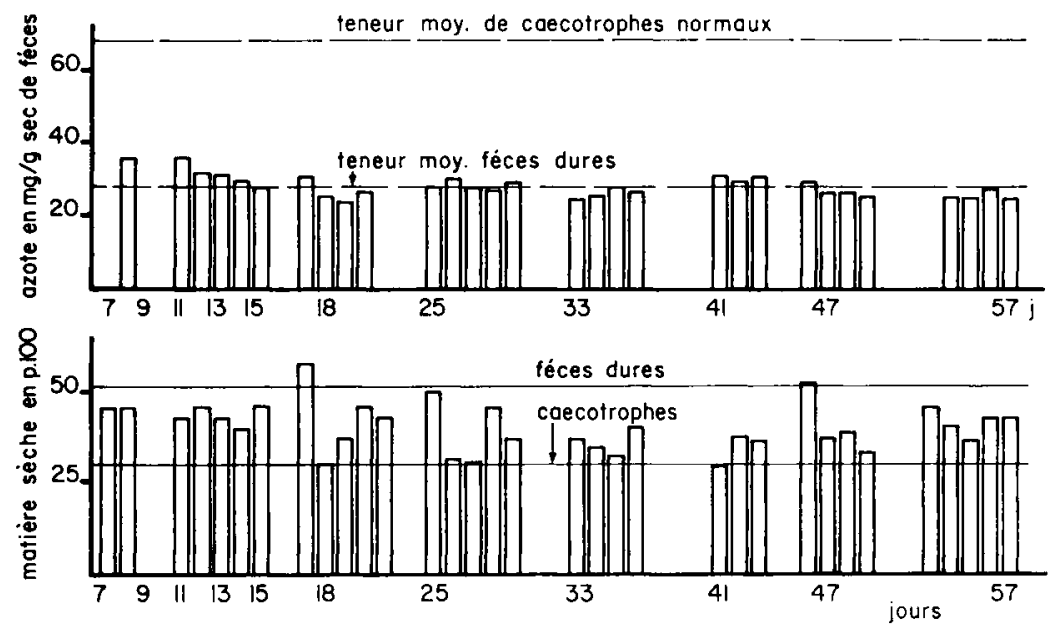

FIG. 2. - Evolution de la teneur en azote ef en matière sèche des fèces produites par un groupe de 6 Lapins après ablation du côlon proximal. La teneur en azote varie peu d'un jour à l'autre et reste voisine de celle de fèces dures normales. La teneur en matière sèche varie entre celle des fèces dures normales et des caecotrophes.

En ce qui concerne les résultats relatifs au rythme d'émission des fèces, rappelons que chez le Lapin normal il existe un rythme circadien, ou nycthéméral de production soit de fèces dures soit de cæcotrophes. Dans nos conditions d'élevage les fèces dures sont produites de $15 \mathrm{~h}$ à $5 \mathrm{~h}$ du matin environ et les cæcotrophes de $6 \mathrm{~h}$ à $14 \mathrm{~h}$. Chez les animaux opérés les fèces sont émises tout au long du nycthémère avec toutefois une nefte diminution de la quantité aux heures qui correspondent à la période normale de la cæcotrophie. Nous avons observé ce phénomène chez tous les Lapins et nous en avons vérifié la persistance en les plaçant plusieurs jours de suife sur le collecteur de fèces (par fraction) avec ou sans le port du carcan.

\section{Discussion.}

La colectomie totale du côlon proximal chez le Lapin supprime de façon définitive la dualité de l'excrétion fécale (durée testée : 2,5 mois). Ces résultats confirment ceux de Bonnafous et Raynaud (1967) et ceux de Hamery (1976) en ce qui concerne le rôle fondamental joué par cette partie du gros intestin dans la formation des cæcotrophes. Nos résultats montrent en outre qu'il n'y a pas de vicariance possible à partir du côlon distal ou du cæcum, tout au moins pendant deux mois et demi.

L'ablation du côlon proximal est une opération relativement traumatisante. En effet les animaux ont des consommations alimentaires faibles et par conséquent la reprise de poids est longue. Toutefois l'intervention n'est pas mortelle. Certains auteurs 
avaient déjà tenté cette même opération et avaient conclu, devant les pertes subies, que cette partie de l'intestin était indispensable à la survie du Lapin. Ces auteurs alimentaient leurs animaux avec de la luzerne et du foin ; par contre les nôtres reçoivent exclusivement un aliment complet équilibré sous forme de granulés. Cette différence de régime peut-elle rendre compte des différences observées au niveau des résultats ?

Cette expérience montre également que la suppression totale de la pratique de la cæcotrophie n'est pas incompatible avec la possibilifé de croissance.

Le tableau relatif à l'évolution pondérale des animaux est intéressant à commenter. Nous constatons en effet que les Lapins de poids voisins de $4,5 \mathrm{~kg}$ ne peuvent pas rattraper leur poids initial à la fin de l'expérience (2,5 mois après) alors que ceux qui ont un poids moyen voisin de $2,8 \mathrm{~kg}$ le retrouvent. Il est difficile de dire sur un nombre aussi faible d'animaux si ce phénomène est généralisable, il sera donc nécessaire de recommencer l'expérience sur un effectif plus grand. Si nous retrouvons cette évolution pondérale différente pour des animaux en croissance ou des adultes cela signifie peut-être que la suppression de la cæcotrophie n'est pas supportée de la même façon par les deux groupes d'animaux, et dans ce cas l'intérêt nutritionnel de ce comportement serait différent selon l'état physiologique du sujet.

En ce qui concerne le rythme d'émission des fèces nous observons le maintien d'une période pendant laquelle peu de « fèces intermédiaires » sont produites. Or cette période correspond précisément à la production des cæcotrophes chez les Lapins normaux. En effet la majorité des auteurs sont d'accord pour situer la cæcotrophie pendant la matinée (Bezille, Gallouin et Le Bars, 1973 ; Laplace, Lebas et Rioperez, 1974 ; Fioramonti et Ruckebusch, 1976). Ce résultat suggère donc que la présence du côlon proximal est bien indispensable pour que les cæcotrophes se forment mais que "l'horloge » qui commande cette différenciation des fèces en est indépendante ef qu'elle n'est pas perturbée par l'intervention chirurgicale.

Dans le chapitre consacré à l'exposé des résultats nous avons souligné le fait que le contenu du cæcum était plus déshydraté que d'habitude. Une interprétation de ce phénomène peut être avancée à la lumière des résultats obtenus par Pickard et Stevens qui concluent à un reflux d'eau du côlon proximal au cæcum. Cet antipéristaltisme a été également souligné par Ruckebusch et Fioramonti (1976) qui observent la présence d'ondes antipéristaltiques sur le côlon proximal dont la naissance ou la propagation dépendrait de l'activité du fusus coli qui jouerait un rôle de pace-maker. Dans notre expérience, l'exérèse totale du côlon proximal, en coupant la voie de conduction en provenance du fusus coli abolit sans doute également ce reflux de l'eau vers le cæcum.

Commission CNERNA Digestion-Absorption/Association des Physiologistes, Paris 5-6 octobre 1978.

\section{Références}

BACQUÈS C., PERRET J. P., 1971. Modifications apportées par la caecumectomie à la répartition et à la nature des constituants lipidiques du contenu digestifs du lapin. Ann. Biol. anim. Bioch. Biophys., 11, 113-127.

BEZILLE P., GALLOUIN F., LE BARS H., 1973. Réalisation d'anus artificiels chez le lapin : mise en évidence du déterminisme et étude du rythme nycthéméral de la caecotrophie. Ann. Biol. anim. Bioch. Biophys., 13, 710-711. 
BONNAFOUS R., RAYNAUD P., 1967. Recherches sur le rôle du côlon dans la dualité de l'excrétion fécale du Japin. Arch. Sci. physiol., 21, 261-279.

FIORAMONTI J., RUCKEBUSCH Y., 1976. La motricité caecale chez le lapin. 3) Dualité de l'excrétion fècale. Ann. Rech. vet., 7, 281-295.

GALLOUIN F., DEMAUX G., LE BARS H., GUEMON L., 1978. Isolement du caecum chez le lapin. $2^{e}$ journ. Rech. Cunicole. Toulouse 4-5 avril, ASFC Paris ed., Commun. ${ }^{\circ} 3$.

HAMERY H., 1976. Mécanisme de la caefrocophie du lapin : essais de colectomie partielle. Thèse Dr. Vét. Alfort 70 pp.

HERNDON J. F., HOVE E. L., 1955. Surgical removal of the caecum and its effect on digestion and growth in rabbits. J. Nutr., 57, 261-270.

LAPLACE J. P., LEBAS F., RIOPEREZ J., 1974. Le transit digestif chez le lapin. 1) Utilisation du Cérium 141 : étude méthodologique et descriptive. Ann. Zootech., 23, 555-576.

PICKARD D. W., STEVENS C. E., 1972. Digesta flow through the rabbit large intestine. Amer. J. Physiol., 222, 1161-1166.

RUCKEBUSCH Y., FIORAMONTI J., 1976. The fusus coli of the rabbit as a pacemaker area. Experientia, 32, 1023-1024. 